\title{
Familial SDHC Mutation Associated With Prolactin/GH-Secreting Pituitary Adenoma and Paraganglioma
}

\author{
Mohammed Barigou', Alexandre Buffet ${ }^{1}$, Antoine Bennet ${ }^{1}$, Pascal Pigny ${ }^{2}$, Laurent Bellec ${ }^{3}$, Philippe Caron ${ }^{1}$, Delphine Vezzosi $^{1}$ \\ ${ }^{1} \mathrm{CHU}$ Larrey, Toulouse, France; ${ }^{2} \mathrm{CHRU}$ de Lille-Lille, France; ${ }^{3} \mathrm{CHU}$ Rangueil-Toulouse, France
}

Introduction: $S D H x$ genes mutations are associated with hereditary pheochromocytoma and paraganglioma syndromes. We describe the very rare case of a patient with $S D H C$ related familial paraganglioma and pituitary adenoma.

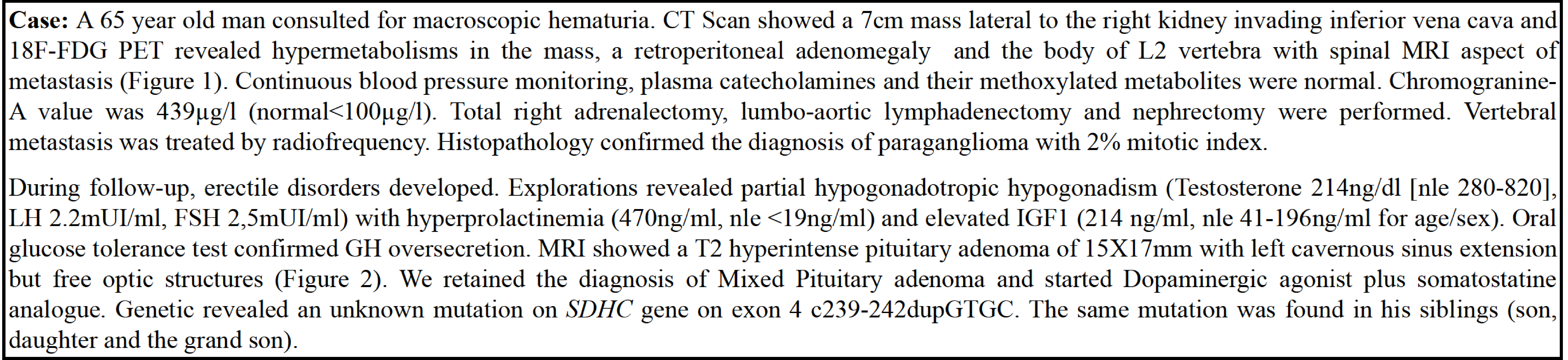

Discussion: $S D H x$ genes mutations are well known causes of familial paraganglioma and pheochromocytoma (1). These mutations alter the mitochondrial complexe 2 , resulting in an increase in $H I F \alpha, V E G F, T G F \alpha$, and $E P O$ that will activate cellular divisions and inactivate apoptosis. Germline $S D H x$ mutations associated pituitary adenoma have been reported sporadically in the literature (2)(3). The specific situation of SDHC mutation associated paraganglioma and pituitary adenoma concerned just one prior case described by Jimenez E \& al (4). Whether $S D H x$ mutations are implicated partially or principally in the neoplastic process of pituitary adenomas is not fully clear. In one case of a bilateral phechromocytoma associated GH secreting pituitary adenoma reported by Xerouki \& al (5), the germinal mutation on SDHD gene (c.298_301delACTC) was associated with a loss of heterozygosity for the $S D H D$ genetic locus and down-regulation of $S D H D$ protein in pituitary adenomas. Recently, two large series including 309 and 80 cases of isolated pituitary adenomas interested on their $S D H A / B$ immunoblotting and immunochemistry an reported a prevalence of abnormal $S D H x$ immunostaining in 0,3 an $3 \%$ of pituitary tumors respectively (1)(6). Most of these cases were macroprolactinomas with higher Ki67 proliferation index compared to normal SDHx staining pituitary adenomas.

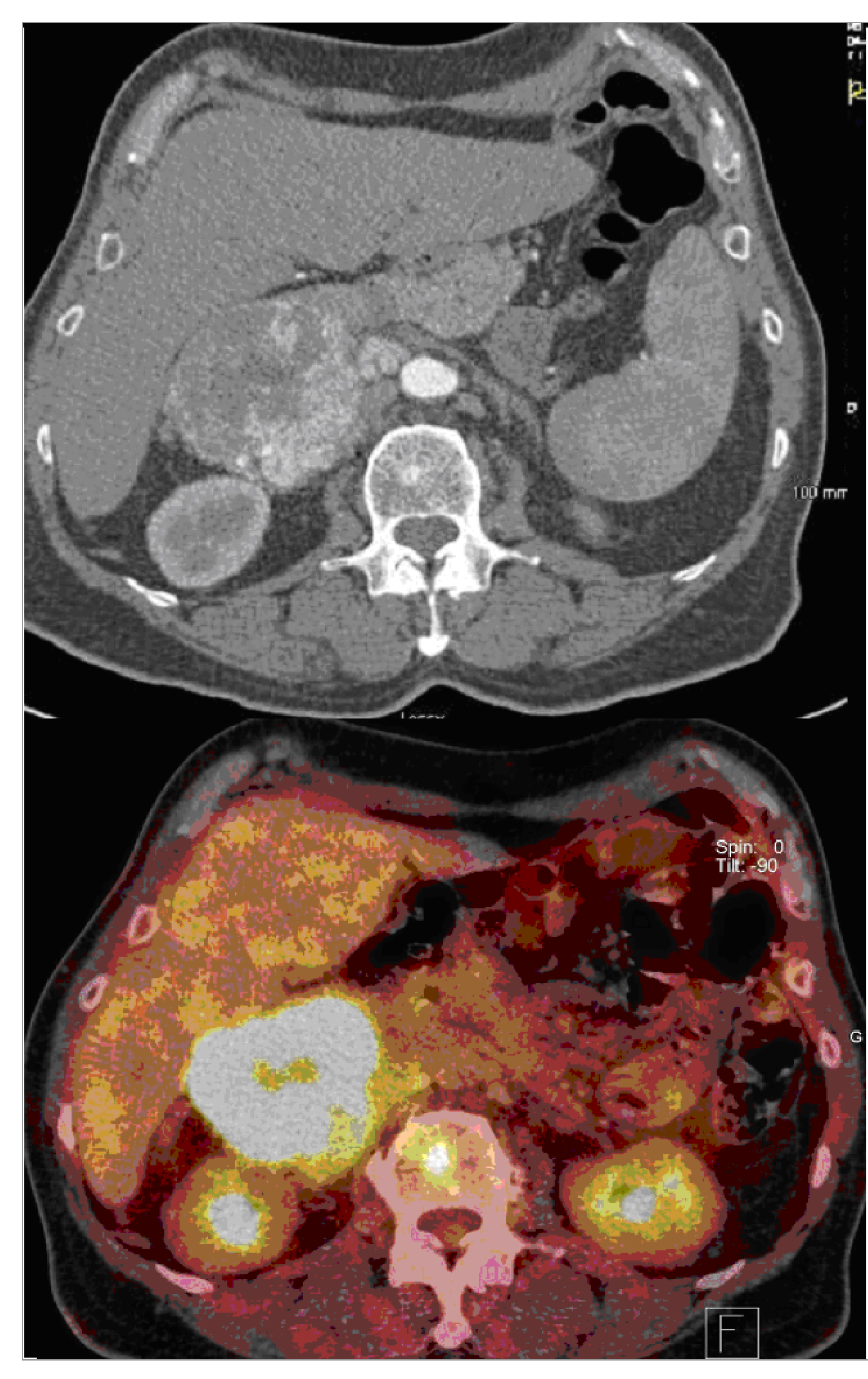

Figure 1: CT scan and PET CT (18FDG) revealing a $7 \mathrm{~cm}$ mass (arrows) located laterally to the right kidney.

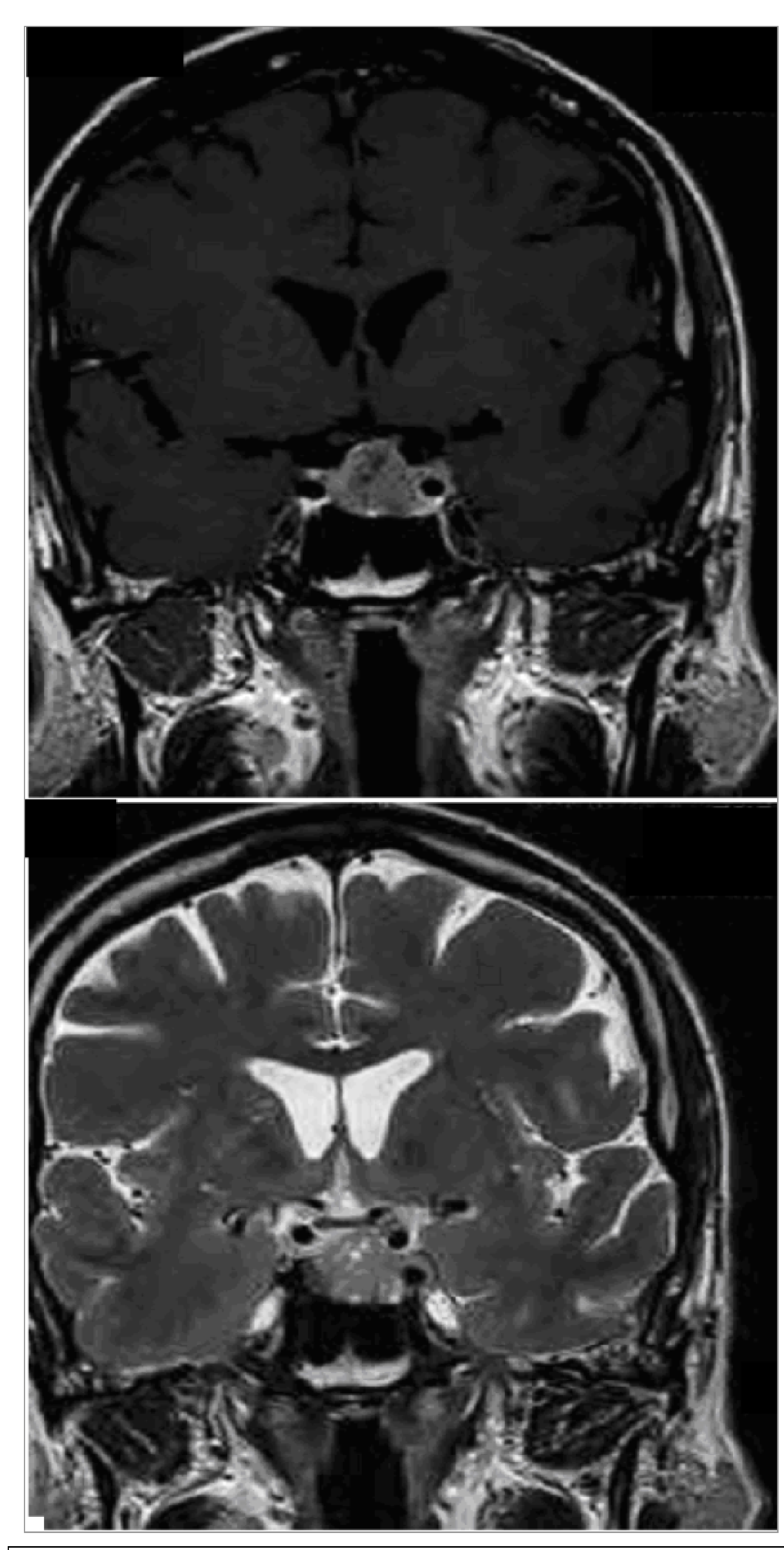

Figure 2: Pituitary MRI revealed an intrasellar lesion measuring $15 \times 17 \mathrm{~mm}$ with T2 hyperintense, T1 hypointense aspect and increased signal after gadolinium infusion compatible with pituitary adenoma.

Conclusion: To our knowledge it is the second reported observation of $\boldsymbol{S D H C}$ mutation associated paraganglioma and pituitary adenoma. This case and the review of the literature suggest that $S D H x$ gene mutation could be, in very rare cases, related to pituitary adenomas occurrence. Our patient was managed by dopaminergic agonist plus somatostatine analogues allowing the control of it's pituitary ovesecretions. The patient benefits of close follow-up for his metastatic pheochromocytoma with stable metastatic disease until now.

\section{References:}

1. Gill AJ, Toon CW, Clarkson A, Sioson L, Chou A, Winship I, et al. Succinate Dehydrogenase Deficiency Is Rare in Pituitary Adenomas. Am J Surg Pathol. 2014 Apr;38(4):560-6.

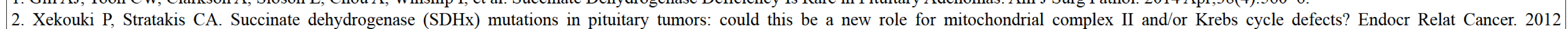
Dec;19(6):C33-40.

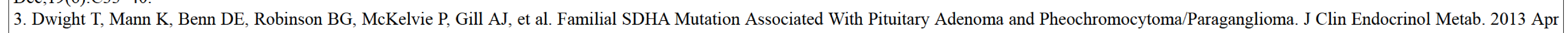
30;98(6):E1103-8.

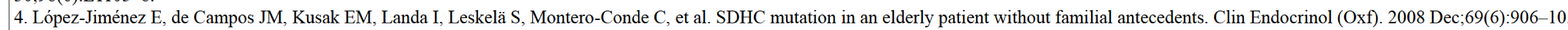

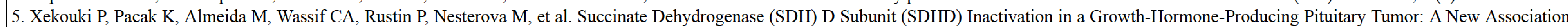
for SDH? J Clin Endocrinol Metab. 2012 Mar;97(3):E357-66.

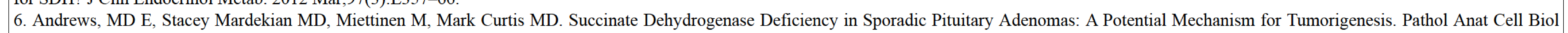
Resid Posters [Internet]. 2014 Jul 1; Available from: http://jdc.jefferson.edu/pacbresidentposters/6

For correspondance: Mohammed Barigou, service d'endocrinologie et maladies métaboliques, Hôpital Larrey 24 chemin de Pouvourville TSA-30030, 31059 Toulouse cedex 9 , France. 